\title{
Polarization properties of superposition states of the quantum transition of molecular iodine vapor in a magnetic field and their possible application
}

\author{
K.Sh. Gazizov, and I.I. Popov** \\ Volga State Technological University, 3 Lenin sq., Yoshkar-Ola, 424001 Russia
}

\begin{abstract}
This paper considers the problem of the possibility of creating a technology for constructing a high-speed high-precision laser lidar based on the physics of the nonFaraday rotation effect of the polarization plane of the primary photon echo in molecular iodine vapor at room temperature. A method is proposed for increasing the range of distance measurements by combining the capabilities of an optical echo-range lidar and an optical time-pulse range lidar.
\end{abstract}

The possibility of creating a technology for constructing a high-precision fast-acting phase-pulse laser range finder (lidar) based on the effect of the non-Faraday rotation of the plane of polarization of the PE in molecular iodine pairs is presented in this paper $[1,2]$.

In the formation of the photon echo the process of creating equally polarized pseudoelectric dipoles happens in all particles simultaneously. After the end of the action of the short pulse, the process of dephasing the dipoles takes place, and then after the arrival of the next pulses - their phase shift. Which leads to the formation of a photon echo signal. At that, on the left-handed and dextrorotatory components of the hyperfine structure of the excited quantum level under the influence of the magnetic field, dephasing (phase shifting) of dipoles takes place at different phase velocities. This is the reason for the non-Faraday rotation of the polarization plane of the photon echo. The magnitude of this rotation depends on the strength of the magnetic field (the magnitude of splitting of the hyperfine components of the excited quantum level) and the time interval between the exciting pulses. It does not depend on the number of resonance particles, that is, on the length of the radiation path in this medium. The magnitude of the angle $\varphi$ of rotation of the PE polarization plane is than 3 times bigger in exponent part than the Faraday rotation at the same value of the applied magnetic field intensity.

Using the dependence of this rotation on the time interval between excitation pulses, we propose a method of high-speed, high-precision distance measurement [3]. In such a laser rangefinder, the first exciting pulse is a reference pulse, and the second is a probing pulse. In this connection the recorded angle of rotation of the echo signal polarization plane contains information about the time of passage of the measured distance in both directions by the probing pulse. This time is recalculated into the measured distance.

The essence of the technology lies in the longitudinal application to the iodine vapor of the required intensity of a homogeneous magnetic field and the provision of longer relaxation time for iodine vapor than the sum of the durations of the exciting pulses and the time interval

\footnotetext{
* Corresponding author: popov@,volgatech.net
} 
separating them. The disadvantage of such a range finder is the limitations on the magnitude of the measured distance, determined by the magnitude of the irreversible transverse relaxation time $T_{2}$.

In this paper it is reported about the conjugation [4] of the above method of measuring distances with a time-pulse method. The first exciting laser pulse is applied to a resonant medium with a strictly fixed time delay in reference to the moment of departure to the distance of the probe pulse. The magnitude of this delay can be any, it corresponds to a strictly defined analytically added (or measured by means of a time-impulse range-finder) distance, to which the additional measurement made with a high precision by the photon echo is added. The sum of the delay time of the reference signal and the time interval measured by the echodistance meter will be the recorded time for the probe pulse to travel two measured distances. The process of measuring the distance is made in 2 stages. At the first stage, the range finder operates in the time-impulse rangefinder mode. Measurement of the distance in this case occurs with an accuracy of one to tens of centimeters (depending on the duration of the exciting pulses). At the second stage, the range finder operates in the phase high-precision range finder mode. In this case, a time interval less than half the relaxation time $T_{2}$ is measured, which is about of $15 \mathrm{~ns}$. This is possible due to the fact that the time of delivery of the first excitation pulse (reference pulse (OI) of the range finder) to the resonant medium is delayed by a time interval equal to the multiplication of the whole number of time intervals equal to half the time $T_{2}$ determined by the time-pulse range finder.

It is to this value that the reference pulse is delayed in order to reduce the time interval between the reference and probing (ZI) pulses of the range finder of relaxation to a value not exceeding the time of relaxation the medium $T_{2}$.

The angle $\varphi$ is recalculated by the formula (1) into the value of the time interval $\tau_{12}$ separating the excitation pulses

$$
\tau_{12}=\frac{2}{144 . \times H} \times \operatorname{arctg} \frac{-2 \pm \sqrt{4+3 \operatorname{tg}^{2} \varphi}}{\operatorname{tg} \varphi}+2 \pi n, \quad n \in Z,
$$

where $\mathrm{Z}$ is the set of whole numbers (1).

The step of additional measuring the distance is chosen equal to 5 microns, which corresponds to the time of its passage by an optical pulse equal to $15 \mathrm{fs}$. The result of the measurement of the distance is summed up with the delay time of the first pulse, given with an accuracy of $15 \mathrm{fs}$, and of the additional distance measurement.

\section{References}

[1] I.I. Popov, I.S. Bikbov, I.V. Evseev, V.V. Samartsev, J. Appl. Spectr. 52(5), 794 (1990)

[2] I.V. Evseev, V.M. Ermachenko, Opt. Spectrosc. 47(6), 1139 (1979)

[3] K.Sh. Gazizov, A.M. Gladyshev, I.I. Popov, V.V. Samartsev, I.S. Bikbov, Patent IPC G01C3/00(2006/01) No. 2306527 (November 7, 2005)

[4] K.Sh. Gazizov, I.I. Popov, and V.T. Sidorova, Physics of Wave Phenomena 20(1), 14 (2012) 